Trauma Berufskrankh $2005 \cdot 7$ [Suppl 2]: S317-S319 DOI 10.1007/s10039-004-0984-4

Online publiziert: 8. Dezember 2004

(c) Springer Medizin Verlag 2004

\section{P. Leucht · I. Marzi}

Klinik für Unfall-, Hand- und Wiederherstellungschirurgie,

Johann-Wolfgang-Goethe-Universität, Frankfurt am Main

\title{
Organkomplikationen bei Verletzungen und Eingriffen an der Rumpfwirbelsäule
}

gesamt wenige repräsentative Publikation vorliegen.

\section{Organkomplikationen \\ bei Verletzungen der Rumpfwirbelsäule}

Überwiegend sind thorakale und abdominale Organe betroffen, welche durch ihre anatomische Nähe zur Rumpfwirbelsäule in Mitleidenschaft gezogen werden.

Ursächlich müssen 2 verschiedene Verletzungsmuster der abdominalen Begleitverletzung unterschieden werden:

- das direkte Stoßtrauma im Sinne eines stumpfen Bauchtraumas sowie

- das Akzelerations-/Dezelerationstrauma, welches indirekt durch Scherkräfte zu einer Organ- oder Gefäßverletzung führt.
Bei Frakturen der Lendenwirbelsäule, die als Last tragendes Element des Achsenskeletts eine hohe Stabilität ihrer knöchernen Strukturen aufweist, wird eine Rate der abdominalen Begleitverletzungen von 3-10\% angegeben [3, 4]. Sie treten sowohl nach direktem Trauma als auch nach Dezelerationsverletzungen auf. Die Ursache für diesen doch relativ niedrigen Wert resultiert aus dem Frakturmechanismus. Allerdings sollte man bezüglich der anatomischen Nähe der Bauchorgane zur LWS und der hohen notwendigen Kraft, die zur Fraktur führt, eine höhere Koinzidenz auch bei isolierten Verletzungen erwarten [5].

\section{Eigene Ergebnisse}

In einer vorläufigen Auswertung eigener Fälle aus den Jahren 2003 und 2004 der Klinik für Unfall-, Hand- und Wiederher-

\footnotetext{
- Milzrupturen

- Leberrupturen

- Mesenterialabrisse

- Pankreaskontusion

- Nierenkontusion

- Zwerchfellrupturen
}

Eine auf diese Thematik bezogene Literaturrecherche ergab nur eine geringe Anzahl von Arbeiten, sodass im Folgenden ein kurzer Überblick über Organverletzungen bei Frakturen der Rumpfwirbelsäule gegeben wird.

Organkomplikationen bei Eingriffen an der Rumpfwirbelsäule weisen unterschiedliche Häufigkeiten je nach Zugangswahl auf. So ist das Risiko der Organverletzung bei der dorsalen Instrumentierung um ein Vielfaches geringer als bei der ventralen Spondylodese. Ebenso muss zwischen peri- und postoperativen Komplikationen unterschieden werden, wobei ins-

Abb. 1 - 48-jährigermännlicher Patient, BWK-12Fraktur mit B-Komponente, ausgeprägtes retroperitoneales Hämatom links, Milzlazeration am kaudalen Pol, Leberlazeration, freie Luft intraperitoneal bei Duodenumruptur, Nierenperfusionsstörung bei Intimaeinriss der A. renalis rechts

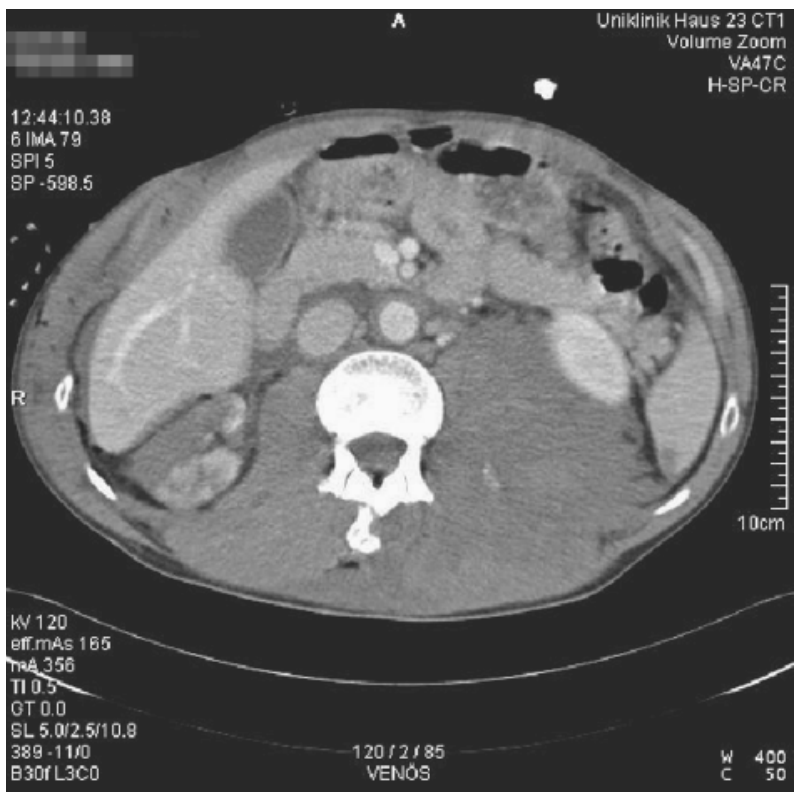




\section{Zusammenfassung · Abstract}

Trauma Berufskrankh 2005 · 7[Suppl 2]:S317-S319 DOI 10.1007/s10039-004-0984-4

๑) Springer Medizin Verlag 2004

P. Leucht $\cdot$ I. Marzi

\section{Organkomplikationen bei Verletzungen und Eingriffen an der Rumpfwirbelsäule}

\section{Zusammenfassung}

Organverletzungen bei Frakturen der Rumpfwirbelsäule können entweder als Folge eines direkten Traumas oder indirekt durch Scherbewegungen bei Hochrasanztraumen entstehen. Indirekte Marker für abdominale Verletzungen sind die Querfortsatzfraktur der Lendenwirbelsäule oder die Rippenserienfraktur bei thorakalen Begleitverletzungen. Organkomplikationen treten bei der operativen Versorgung der Rumpfwirbelsäulenfraktur in der dorsalen Technik nur ausnahmsweise auf. Der ventrale Zugang kann zu einer Verletzung aller intrathorakalen oder intraabdominalen Organe führen, was operationstaktisch berücksichtigt werden muss.

\section{Schlüsselwörter}

Organkomplikationen · Frakturen . Operative Versorgung $\cdot$ LWS · BWS

\section{Organ complications following injuries and surgery in the thoracic and lumbar segments of the spine}

\begin{abstract}
Organ injuries in the presence of thoracolumbar spinal fractures can result from a direct blunt trauma or arise indirectly from strong shearing forces in the case of highimpact trauma. Transverse process fracture in the lumbar spine should be regarded as a significant marker for injuries of abdominal organs, as well as the multiple rib fracture for the thoracic organ injury. Organ complications due to posterior surgery of the thoracolumbar spine is very rare. Using the anterior approach, every abdominal or thoracic organ may be injured and has to be considered during surgery.
\end{abstract}

\section{Keywords}

Organ complications · Fractures .

Spine surgery - Thoracolumbar spine

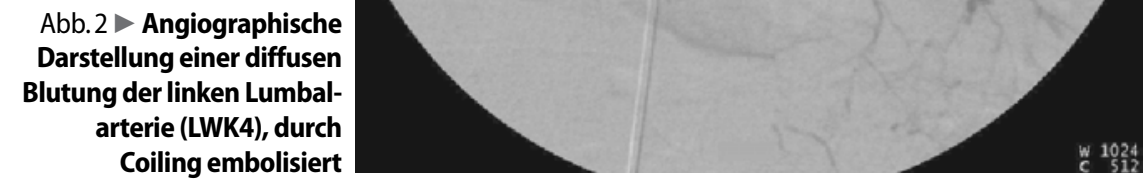

stellungschirurgie der Johann-WolfgangGoethe-Universität Frankfurt am Main konnten 118 Patienten mit einer Verletzung der Brust- oder Lendenwirbelsäule berücksichtigt werden. Die 3 häufigsten Unfallursachen waren

\section{- Verkehrsunfall mit dem PKW \\ - Sturz aus großer Höhe \\ - banaler Sturz}

In 10 Fällen wurde ein kompletter motorischer und sensorischer Querschnitt diagnostiziert, 9 Patienten litten an einem inkompletten Querschnitt, und 95 Patienten zeigten keine neurologischen Ausfallerscheinungen. In diesem Kollektiv fanden sich 58 thorakale und 68 abdominale Begleitverletzungen. Insgesamt wurden 32 Extremitätenverletzungen und 30 Verletzungen des Schädels festgestellt.

Dieses Kollektiv weist eine deutliche Häufung von Hochrasanztraumen auf, wodurch die relativ hohe Rate von 49,2\% thorakaler und 57,6\% abdominaler Begleitverletzungen erklärt werden kann. Die am häufigsten betroffenen Organe waren in absteigender Reihenfolge Milz, Niere, Nebenniere, Leber, Dünndarm und Mesenterium. Tendenziell ließ sich die Nierenverletzung häufiger bei Frakturen der kranialen Lendenwirbelsäule nachweisen. Dies ist zum einen auf die räumliche Nähe, anderseits auf das Trauma, welches in diesem Fall auf den unteren Rippenbogen zentriert ist, zurückzuführen. Im Gegensatz dazu waren Milz, trotz ihrer anatomischen Lage, und Dünndarm vorwie- gend bei Verletzungen der unteren LWS mitbetroffen. Leberverletzungen im Sinne von Lazerationen oder Rupturen wiesen keinen direkten Zusammenhang mit der Frakturhöhe auf (• Abb. 1, 2).

Die Mehretagenfraktur der Lendenwirbelsäule ging mit einer höheren Rate an abdominalen Begleitverletzungen einher. Zu erklären ist dies durch eine höhere Krafteinwirkung, welche die Grundlage für eine Mehretagenfraktur bildet.

\section{LWS-Hochrasanztraumen und abdominale Begleitverletzungen}

Wie oben beschrieben, werden die Frakturen der lumbalen Wirbelsäule vorwiegend durch Kompressions-, Flexions-/ Distraktions- und Rotationskräfte ausgelöst. Miller et al. [2] untersuchten ein Kollektiv von 191 Patienten mit Frakturen der Lendenwirbelsäule auf das Zusammenspiel von Processus-transversus-Fraktur und Bauchorganverletzung. Sie kamen zu dem Ergebnis, dass es einen signifikanten Zusammenhang gibt. So hatten $48 \%$ der Patienten mit Querfortsatzfraktur eine abdominale Begleitverletzung, während die Patienten ohne Querfortsatzfraktur nur in $6 \%$ an Bauchorganverletzungen litten.

Die Erklärung liegt auch hier in der Energie des Unfalls. An den Querfortsätzen der Lendenwirbelsäule setzen der M. quadratus lumborum und der M. psoas an. Kommt es zu einem Hochrasanztrauma, wirken durch den Zug dieser beiden Muskeln immense Kräfte auf den Querfortsatz ein, die dann in einer Frak- 
tur enden. Überwiegend handelt es sich hierbei um Flexions-/Extensions- und Rotationskräfte. Unterstützt wird diese Erklärung dadurch, dass in diesem Kollektiv keine Processus-transversus-Fraktur durch ein stumpfes Stoßtrauma initiiert wurde.

Die Querfortsatzfraktur darf also nicht wie bisher auf die gleiche Schweregradsstufe wie die Pedikel- oder Bogenfraktur gestellt werden. Vielmehr sollte sie den Untersucher, als indirekter Marker, auf ein Hochrasanztrauma hinweisen, welches auslösend für eine intraabdominale Verletzung sein kann.

\section{BWS-Hochrasanztraumen und thorakale Begleitverletzungen}

Thorakale Begleitverletzungen beschränken sich im Wesentlichen auf Verletzungen der Lunge, des Herzens und der angrenzenden Gefäße. In einem 1-Jahres-Zeitraum von 2002-2003 wurden im Schockraum der Klinik für Unfall-, Hand- und Wiederherstellungschirurgie der Johann-Wolfgang-Goethe-Universität Frankfurt am Main 411 Patienten behandelt. In 26 Fällen wurde eine Verletzung der Wirbelsäule diagnostiziert. Hiervon waren 21 Patienten polytraumatisiert, 14 hatten eine thorakale, 5 eine abdominale Begleitverletzung erlitten. Die hohe Rate an thorakalen Begleitverletzungen ist mit der engen anatomischen Beziehung der Wirbelsäule und der intrathorakalen Organe zu erklären. Außerdem fehlt dem Thorax, aufgrund seiner relativ rigiden Ummantelung durch die Rippen, im Gegensatz zum Abdomen die notwendige Elastizität, um die einwirkende Kraft zu neutralisieren.

Ähnlich der Processus-transversusFraktur der Lendenwirbelsäule muss die wirbelsäulennahe Rippenserienfraktur als indirekter Marker gewertet werden. Zusätzlich sollte immer auf eine direkte Verletzung des Lungenparenchyms oder der großen Gefäße durch Rippenfragmente geachtet werden.

\section{Organkomplikationen bei Eingriffen an der Rumpfwirbelsäule}

Hier muss hier eine klare Trennung zwischen den Zugangsmöglichkeiten zur Rumpfwirbelsäule getroffen werden.

\section{Dorsaler Zugang}

Der dorsale Zugang zur Implantation eines Fixateur-interne-Systems ist mit einer zu vernachlässigenden Wahrscheinlichkeit der Organkomplikation behaftet. Lediglich die ventrale Perforation der Pedikelschrauben kann zu einer Verletzung der Aorta oder V. cava, die der Wirbelsäule direkt aufliegen, führen. Hier sind insbesondere das sorgfältige Austasten der Pedikel und in $\mathrm{Zu}$ kunft ggf. die navigierte Operation mit dreidimensionaler Bildgebung von Bedeutung.

\section{Ventraler Zugang}

Er kann sowohl konventionell offen als auch minimalinvasiv gewählt werden. Hier ist eine Organverletzung wahrscheinlicher. Dennoch führte eine Medline-Suche nur zu einer geringen Anzahl verwertbarer Artikel.

Die Multicenter-Studie der AG Wirbelsäule fand in ihrem Kollektiv von 682 Patienten $4(0,6 \%)$ abdominale und $5(0,7 \%)$ pulmonale Komplikationen. Diese entstandenjedoch nicht direkt durch das chirurgische Vorgehen, sondern waren vielmehr Ausdruck einer systemischen Reaktion auf die Verletzungsschwere. Die pulmonalen Komplikationen waren in 2 Fällen durch eine Pneumonie, in 2 weiteren Fällen durch eine respiratorische Insuffizienz bedingt. Ein Patient erhielt eine Pleuradränage bei postoperativem Erguss. Bei den abdominalen Komplikationen handelte es sich bei 2 Patienten um einen Ileus, 1 Patient erlitt eine Ulkusblutung, 1 weiterer Patient entwickelte eine Pankreatitis bei stumpfem Bauchtrauma [1].

Typische intraoperative Komplikationen des ventralen thorakalen Zugangs sind die Verletzung des Lungenparenchyms oder der Milz bei Mobilisation des Zwerchfells zur Versorgung tief liegender thorakaler oder hoher lumbaler Frakturen. Die Verletzung von Blut- und Lymphgefäßen kann zum Hämato- bzw. Chylothorax führen.

\section{Retroperitonealer Zugang}

Er dient zur Versorgung der lumbalen Wirbelfrakturen und kann theoretisch $\mathrm{zu}$ einer Verletzung aller intraabdominalen Organe führen. Häufig sind Verletzungen der Ureteren, Gefäße und Nerven. Insbesondere die Läsion des N. genitofemoralis bei der Mobilisation des M. psoas sollte erwähnt werden.

\section{Langzeitkomplikationen}

Sie sind von obigen, bereits intraoperativ aufgetretenen Komplikationen abzugrenzen. Hierunter finden sich z. B. die Bauchwandhernie oder die Affektion durch den Organen direkt anliegendes Osteosynthesematerial. So ist von einem Patienten aus dem eigenen Krankengut zu berichten, welcher bei persistierender Schmerzsymptomatik auf der Entfernung des ventralen Implantats bestand. Bei der Lösung des ventralen Plattensystems kam es zur vollständigen Perforation einer Aortenarrosion, die nur durch ein kurzstreckiges Gefäßinterponat beherrscht werden konnte. Die stetige Pulswelle im Kontakt mit dem kranialen Anteil des Plattensystems hatte zu einem Druckulkus der Aortenwand geführt, welches in absehbarer Zeit spontan perforiert wäre.

\section{Resümee}

Trotz einer Dunkelziffer muss von einer geringen Rate an schwer wiegenden Komplikationen durch die operative Wirbelsäulenbehandlung ausgegangen werden [6].

\section{Korrespondierender Autor}

\section{P. Leucht}

Universitätsklinik für Unfall-, Hand- und Wiederherstellungschirurgie,

Theodor-Stern-Kai 7, 60590 Frankfurt am Main

E-Mail: P.Leucht@trauma.uni-frankfurt.de

Interessenkonflikt: Keine Angaben

\section{Literatur}

1. Knop C, Blauth M, Bühren V et al. (2000) Operative Behandlung von Verletzungen des thorakolumbalen Überganges. Teil 2: Operation und röntgenologische Befunde. Unfallchirurg 103: 1032-1047

2. Miller CD, Blyth P, Civil IDS (2000) Lumbar transverse process fractures - a sentinel marker of abdominal organ injury. Injury Int J Care Injured 31: 773-776

3. Rabinovici R, Ovadia P, Mathiak G et al. (1999) Abdominal injuries associated with lumbar spine fractures in blunt trauma. Injury Int J Care Injured 30: 471-474

4. Saboe LA, Reid DC, Davis LA et al. (1991) Spine trauma and associated injuries. J Trauma 31: 43-48

5. Sturm JT, Perry JF Jr (1984) Injuries associated with fractures of the transverse processes of the thoracic and lumbar vertebrae. J Trauma 24: 597

6. Verlaan JJ, Diekerhof CH, Buskens E et al. (2004) Surgical treatment of traumatic fractures of the thoracic and lumbar spine. Spine 29: 803-814 